JoHN ILIFFe

\title{
A modern History of Tanganyika
}

African Studies Series 25. Cambridge University Press, Cambridge, London, New York, Melbourne 1979, XVI, 616 S.

Cranford Pratt

The critical Phase in Tanzania 1945-1968

Nyerere and the Emergence of a Socialist Strategy

Cambridge University Press, Cambridge, London, New York, Melbourne 1976, X, 309 S.

Kein politisches System und keine Verfassung kann ertragreich analysiert werden, ohne daß die historische Entwicklung eingehend untersucht wird, die der aktuellen Konstitution eines Gemeinwesens vorausgegangen ist. Dieser als selbstverständlich erscheinende Grundsatz ist bei der Untersuchung des Verfassungsrechts und der Verfassungswirklichkeit schwarzafrikanischer Staaten bisher kaum ausreichend beachtet worden. Autoren aus westlichen Industrieländern haben sich jedenfalls überwiegend darauf beschränkt, die Entwicklung der unabhängigen Staaten Schwarzafrikas vor dem Hintergrund der Kolonialgeschichte zu diskutieren.

Tansania ist ein besonderes plastisches Beispiel für die Unzulänglichkeit dieses Ansatzes. Nyerere rekurriert in seiner politischen Philosophie immer wieder auf Werte, die in den vorkolonialen Gesellschaften Tanganyikas verbreitet gewesen seien und auf die es sich zurückzubesinnen gelte. Nur eine Erforschung der Struktur dieser staatenlosen Gesellschaften vor der Ankunft weißer Missionare und Händler kann Aufschluß darüber geben, ob solche Werte und Verhaltensregeln verbreitet gewesen sind oder ob es sich bei der Aussage Nyereres um ein zwar legitimes, aber historisch nicht zu untermauerndes politisches Postulat auf dem Weg zu einer besseren Gesellschaftsordnung in Tansania handelt.

Der britische Historiker John Iliffe hat nun eine „,Moderne Geschichte Tanganyikas” vorgelegt, deren Titel bereits verdeutlicht, daß dieses Land eine lange Geschichte hinter sich hatte, als Weiße es erstmals betraten. Iliffe, der zuvor bereits mit Arbeiten zur deutschen Kolonialherrschaft in Tanganyika hervorgetreten ist, legt $\mathrm{z}$ war thematisch auch in dieser eingehenden Untersuchung den Schwerpunkt auf die - deutsche und britische - Kolonialgeschichte. Zugleich betont er aber die grundlegende Bedeutung der Geschichte vorkolonialer afrikanischer Gesellschaften, wie sie in neuerer Zeit vor allem von Afrikanern geschrieben wird (S. 3 f.). Iliffe versteht sowohl die Entstehung als auch die Auflösung der Kolonie Tanganyika als dialektischen Prozeß: die koloniale Gesellschaft baute auf vorkolonialen Traditionen auf, und der Nationalismus, der Tanganyika in die Unabhängigkeit führte, knüpfte seinerseits an die Strukturen der kolonialen Gesellschaft an und übernahm sie zum Teil (S. 4). Um so bedeutsamer ist Nyereres direkter Zugriff auf soziale Wertvorstellungen aus der vorkolonialen Zeit, die durch das während der Kolonialherrschaft verstärkt geförderte individuelle Besitzstreben zerstört zu werden drohen.

Der Autor stellt selbst die vorkoloniale Geschichte Tanganyikas nur verhältnismäßig kurz dar, wobei er sich im wesentlichen auf das 19. Jahrhundert beschränkt (Kap. 2 u. 3). Das umfangreiche Quellenmaterial zur deutschen Kolonialherrschaft wertet er dagegen detailliert und differenziert aus. Interessant ist insbesondere sein Versuch, die Okonomie und die Okologie der Kolonialherrschaft zueinander in Beziehung zu setzen (Kap. 5). In einem besonders gut gelungenen Kapitel mit dem überraschenden Titel „The creation of tribes“ (Kap. 10) analysiert Iliffe das Konzept der indirect rule, die von den Briten von Nigeria auf 
Tanganyika übertragen wurde, ohne daß bemerkt worden wäre, daß im vorkolonialen Tanganyika - anders als in Nigeria - nur sehr wenige Gesellschaften als Stämme organisiert waren. Die paternalistische britische Kolonialpolitik grenzte sich von der vorangegangenen repressiveren Politik der Deutschen dadurch ab, daß sie irrtümlich für traditionell gehaltene Herrschaftsstrukturen schuf und für ihre Zwecke benutzte. Die Afrikaner begannen erst daraufhin, ein stärkeres Stammesbewußtsein zu entwickeln, das jedoch zu keiner Zeit stark genug wurde, um die nationalistische Unabhängigkeitsbewegung zu behindern.

Abschließend stellt Iliffe die Entwicklung vom zweiten Weltkrieg bis zur. Unabhängigkeit im Jahre 1961 dar, wobei er der Gewerkschaftsbewegung und der Entstehung der TANU besondere Aufmerksamkeit widmet. In zahlreichen Passagen behandelt Iliffe aber nicht nur die ökonomische, sondern auch die kulturelle Entwicklung Tanganyikas und analysiert z. B. eingehend die Rolle der Religion in verschiedenen historischen Phasen. Der Charakter eines richtungweisenden Handbuchs, den dieser Band in Anspruch nehmen kann, wird dadurch bestätigt.

Drei Jahre vor Iliffe hat der Kanadier Cranford Pratt sein Buch über die Entwicklung Tansanias von 1945 bis zur Arusha Declaration veröffentlicht. Pratt, der ebenso wie Iliffe längere Zeit in Dar es Salaam gelehrt hat, brauchte sich nicht auf geschriebene Quellen zu beschränken, sondern konnte mehrere Gespräche verwerten, die er selbst mit Nyerere und anderen führenden Politikern über die jüngste Vergangenheit Tansanias geführt hatte.

Im ersten Drittel des Buches untersucht Pratt eingehend die britische Politik in Tanganyika seit 1945. Dabei beurteilt er im Gegensatz zu Iliffe das Konzept der indirect rule etwas zu wohlwollend und kritisiert die Briten gerade deswegen, weil sie in den ersten zehn Jahren nach dem 2. Weltkrieg die Methode der indirect rule zugunsten aggressiverer Verwaltungspraktiken und offener Gewaltanwendung aufgegeben hätten (S. 25). Damit stärkten die Kolonialherren unwillkürlich die neugegründete TANU. Der Úbergang zur Unabhängigkeit selbst verlief dagegen gewaltfrei und für britisches Verständnis mustergültig, was z. T. auf das gute persönliche Verhältnis zwischen Nyerere und dem letzten Generalgouverneur Turnbull zurückzuführen ist.

Die besondere Bedeutung der Prattschen Untersuchung ergibt sich jedoch erst aus ihrem zweiten Teil (, Nyerere and the emergence of a democratic and socialist strategy“), deren Titel sich nur unwesentlich vom Untertitel des Buches unterscheidet. Hier wird die politische Entwicklung Tansanias von der Unabhängigkeit bis zur Arusha Declaration mit einer Detailkenntnis analysiert, die deutlich werden läßt, daß hier ein Wissenschaftler den politischen Prozeß engagiert und aus nächster Nähe beobachtet hat. Obwohl Nyerere diese Entwicklung so ausschlaggebend geprägt hat und seine Entscheidungen sowie die zugrunde liegende Vorstellung von einer idealen Gesellschaft daher naturgemäß im Vordergrund stehen, macht Pratt deutlich, wie vielen widersprüchlichen Kräften er in der TANU und in der Bürokratie gegenübergestanden hat, wie er zu Kompromissen genötigt wurde und sich durch seinen moralischen Rigorismus in Einzelfällen auch zu ungerechtfertigten Maßnahmen hat hinreißen lassen. Hier wird also kein geschöntes, sondern ein differenziertes und überzeugendes Bild dieses Staatsmannes gezeichnet.

Die eingangs erwähnte These Nyereres, es gelte, vorkoloniale afrikanische Werte im Bewußtsein der Massen zu stärken, die die Grundlage für einen tansanischen Sozialismus bilden können, ist nach Auffassung Pratts zwar nicht beweisbar, aber für sie spreche doch der Beweis des ersten Anscheins (S. 239).

Wie der langjährige Wirtschaftsberater Nyereres sieht auch Pratt in einer Verschlechterung der wirtschaftlichen Lage, die jetzt durch den anhaltenden Einsatz tansanischer Truppen in Uganda noch verschärft wird, die entscheidende Bedrohung für eine sozialistische Politik. 
Einer Gruppe marxistischer Sozialwissenschaftler in Tansania wirft Pratt vor, sie unterschätze diese Gefahr und messe demgegenüber der Herausbildung einer neuen Bürokratenklasse zu große Bedeutung bei (S. 248). Auch wenn die Auseinandersetzung mit der marxistischen Kritik an Nyerere in einzelnen Punkten differenzierter hätte ausfallen können, hat Pratt mit seiner materialreichen und brillant geschriebenen Untersuchung einen grundlegenden Beitrag zur Analyse des tansanischen Sozialismus geleistet. Man wird sogar John Iliffe zustimmen können, der dieses Buch als das beste bezeichnet hat, das bisher über die politische Entwicklung in Tansania unmittelbar vor und nach der Unabhängigkeit geschrieben worden ist.

Alexander Dix

\section{FRANZ TAESCHNER}

\section{Zünfte und Bruderschaften im Islam}

Texte zur Geschichte der Futuwwa

Bibliothek des Morgenlandes, Artemis Verlag, Zürich, München, 1979, 672 S., 95,- DM

Dieser Band schließt eine Lücke der orientalischen Forschung, da in ihm erstmals umfassend Materialien über Zünfte und Bruderschaften im Islam zusammengetragen und mit erläuternden Ausführungen des Verfassers versehen werden.

Die Futuwwa, abgeleitet von dem arabischen Verbum: jung sein, ist ein weiter Begriff, der die Gesamtheit der edlen, ritterlichen Tugenden des Mannes umfaßt und in der Literatur teilweise als islamisches Rittertum des Mittelalters bezeichnet wird. Der Begriff geht auf altarabische Tradition zurück und wird in islamischer Zeit religiös motiviert.

Die ersten Futuwwa-Gruppen treten als Kampfgruppen auf, sie rauben, morden, ehebrechen und verbreiten durch ihre Gewalttätigkeit Schrecken. Erst vom 12. Jh. an unter dem Abbasiden - Kalif an-Nasir li-Dīn Allah gebärden sie sich disziplinierter und entwickeln eigene Riten für die Aufnahme von Mitgliedern, die der Verfasser mit der Úbersetzung des Hauptwerkes der Futuwwa (kitāb al-futuwwa von Ibn al-Mi'mar) über Organisation und Brauchtum wiedergibt.

Die höf ische Futuwwa endet mit der Eroberung Baghdads (1258 n. Chr.) durch die Mongolen. Sie lebt in Ägypten unter den Mamluken kurzfristig wieder auf, aber auch dort erlischt allmählich das Interesse, es kommt zu häufigen Mißbräuchen und Entartungen, die schließlich dazu führen, daß die gesamte Institution als böse Neuerung (bid'a) verworfen wird. Noch heute ist in Ägypten ein Raufbold ein ,,fetewwa“. Im Irak lebt der Begriff futuwwa bis vor dem 2. Weltkrieg fort und bezeichnet im wesentlichen Organisationen zur vormilitärischen Erziehung der Jugend. In der Türkei überlebt die höfische futuwwa als Achitum ((ahiliq) und mündet schließlich in das Zunftwesen ein. Dieser Entwicklung - namentlich im Osmanischen Reich - sind die beiden letzten Kapitel des Werkes von Taeschner mit zahlreichen übersetzten Texten gewidmet.

Der Achi ist der Vorsteher einer zunftähnlichen Gemeinschaft von Angehörigen des Handwerkerstandes, eine Spezifizierung nach einzelnen Handwerksständen ist zur Zeit der Entstehung des Achitum nicht vorhanden, sie bildet sich - wie die Zunft der Gerber - erst später heraus. Die Hauptrolle des Achitum liegt auf politischem Gebiet, der Achi vertritt den Sultan bzw. Emir in weit vom Herrschaftszentrum entfernten Gebieten. Dem Achischrifttum sind konkrete Angaben über das Bundeswesen zu entnehmen, so die Einteilung der Mitglieder in verschiedenen Klassen (qauli und saifi). Die sogenannte Vogelrede des Gülšehri gibt Auskunft über Themen der sufischen Ethik und weist auf die Affinität des Achitum zum ši'itischen Islam hin. Die Erläuterungen, die der Wiedehopf in dieser Rede den an- 